\title{
THE ESO MEASURING MACHINES
}

\author{
P. GROSBØL \\ European Southern Observatory \\ Karl-Schwarzschild-Straße 2 \\ D-85748 Garching \\ Germany
}

\section{Introduction}

The start of a general plate measuring facility at ESO was made in 1974 with the purchase of an OPTRONICS S-3000 machine placed at the ESO headquarters in Geneva. The facility was complemented around 1977 by a one-dimensional GRANT Series 800 Comparator previously used by ESO Santiago and finally by the acquisition of a PDS 1010A microdensitometer installed in 1979. After the ESO headquarters were moved to Munich in 1980, the measuring machines were reinstalled in the basement close to the ESO plate vault. Due to the declining usage of Coude plates, the GRANT machine was taken out of service in 1988.

\section{Measuring Machine Facility}

The computer and control systems were totally exchanged during the last years to introduce modern technology and thereby minimize maintenance in the future. A central SPARC-10 UNIX system provides general services such as disk storage for data, $\mathrm{I} / \mathrm{O}$-devices and printer to both machines. The server is connected to the machines and the general ESO Local Area Network through Ethernet using TCP/IP protocols. All data acquired are stored on the server disks in either FITS or MIDAS format where they can be analyzed and displayed with MIDAS. This can be done either on a local X11-terminal or from any workstation in the ESO network. Users can then copy their data files to tape (i.e. DDS/DAT or Exabyte-8500) or to their own disk area with the ftp utility. The general layout of the system is shown in Fig. 1.

The new control systems are based on the VME-bus and are virtually identical for the two machines. The CPU, memory and communication boards are off-the-shelf items while a special set of 3 boards designed by RGO is used for the control of the stage and user control panel. The software is written in ANSI-C and used the VxWorks real time kernel. The commands for the two systems are identical, and have a syntax very similar to that of MIDAS. It is also possible to perform a remote login from the control terminal to get information on the network e.g. table of reference stars through the STARCAT interface. 


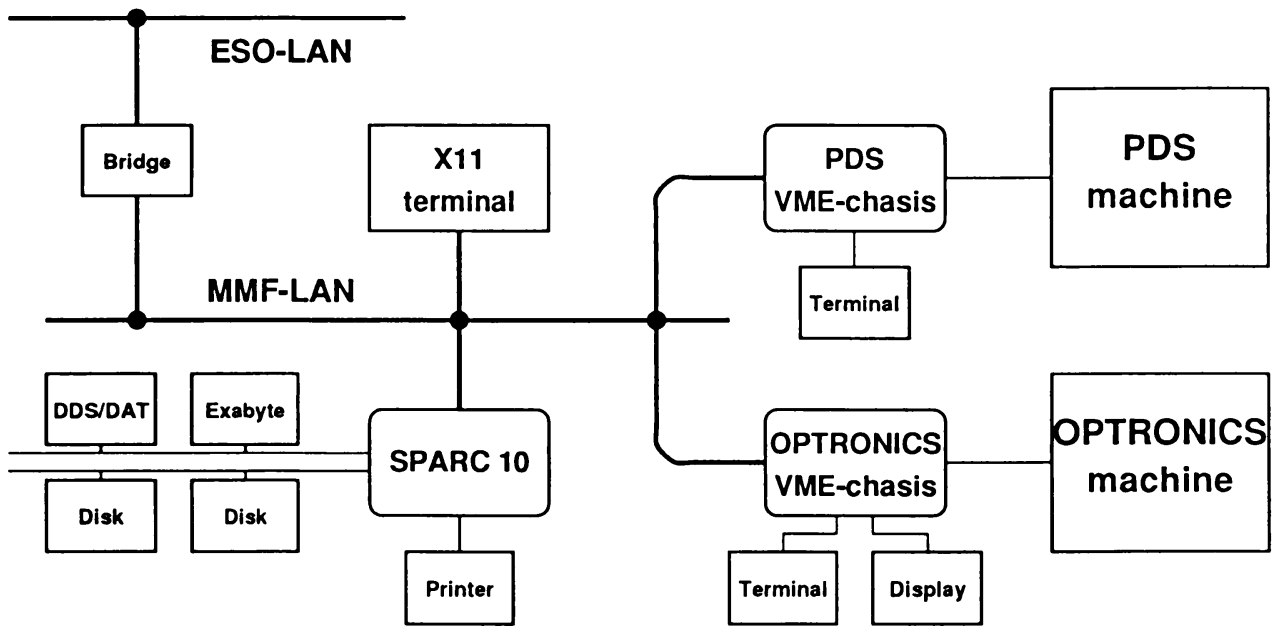

Figure 1. Layout of the measuring machine facility.

The main characteristics of the machines are given as follows:

OPTRONICS: The OPTRONICS S-3000 is a flat bed microdensitometer with a granite base and a stage moving on air bearings. It is mainly used for astrometric measurements. The positional accuracy is about $1 \mu \mathrm{m}$. The stage can be moved over an area of $35 \times 35 \mathrm{~cm}^{2}$ and has a number of adaptors for different plate sizes up to $36 \times 36 \mathrm{~cm}^{2}$ (i.e. Palomar Schmidt plates). The machine has a Full Field CCD camera which covers an area of $3 \times 2 \mathrm{~mm}^{2}$ on the plate. Images from this camera can be transferred to the central server and used as e.g. finding charts. A general scanning option has been foreseen in the design but its implementation has not been possible due to limited man-power.

PDS: The PDS 1010A microdensitometer has a low inertia stage capable of moving with a speed of up to $50 \mathrm{~mm} / \mathrm{sec}$. It is possible to move the stage over an area of approximately $23 \times 23 \mathrm{~cm}^{2}$ but larger plates can be mounted. Square apertures from $5 \mu \mathrm{m}$ to $50 \mu \mathrm{m}$ are available in addition to a number of slits. The effective dynamic range of the analog system depends on the scanning speed used. At low speed, densities in the range 4 - 5 can be measured reliably using the Wisconsin logarithmic amplifier and a 15-bit A/D converter. The mean positional error is of the order of $2 \mu \mathrm{m}$ for both axes. The PDS is recommended for measuring plates with high dynamic range. Also programs which require a large number of stage motions should be executed on the PDS.

\section{Availability}

The ESO Measuring Machine Facility is an open user facility where astronomers can come to measure their plate material. Anybody can apply for time on the machines, although priority is given to people working in the ESO member states and analyzing material obtained at ESO. Visitors are provided with an introduction and manuals but are expected to perform the actual measurements themselves since operator assistance is not available. 